\title{
STEMI in Young Befogged by Aluminum Phosphide Toxicity-Role of ECMO as Salvage Therapy and Trimetazidine and Magnesium to Suppress Arrhythmias
}

\author{
Chaitra C Rao ${ }^{1}$, Gunavanthi Jayakumar Himaaldev²
}

\begin{abstract}
Introduction: Aluminum phosphide poisoning (ALP) has a high-mortality rate despite intensive care management, primarily because it causes severe myocardial depression. This case report highlights the subset of ALP patients presenting as ST elevation myocardial infarction (STEMI) with profound myocardial dysfunction and multiorgan failure and successfully treated with extracorporeal membrane oxygenation (ECMO), trimetazidine, and magnesium.

Case description: A 25-year-old man without any comorbidities was brought to emergency department with dyspnea and hypotension. His electrocardiograph (ECG) revealed STEMI with elevated troponin levels, arterial blood gas (ABG) showed severe metabolic acidosis, and echocardiography (echo) revealed ejection fraction 15\%. He was initiated on venoarterial (VA) ECMO in view of refractory hypotension. History of consumption of three tabs of celphos was revealed later by the family members. He progressed to cardiogenic shock, arrhythmias, respiratory failure, acute kidney injury with severe lactic acidosis, liver injury, pancreatitis, and disseminated intravascular coagulation (DIC). He was successfully supported by ECMO, hemodialysis, magnesium, trimetazidine, $N$-acetyl cysteine, inotropes, and blood products. He was weaned off ECMO on day 6 and was discharged home on day 12. Despite his severe and confounding clinical presentation, he had complete normalization of end-organ dysfunction with no neurological sequela. This case demonstrates the high index of suspicion required for ALP, given the potential for rapid progression and severe multiorgan toxicity. This report also highlights the importance of early referral to a tertiary care center with ECMO capability and also the role of magnesium and trimetazidine to suppress arrhythmias.

Conclusion: Aluminum phosphide poisoning can present as STEMI with cardiogenic shock resulting in acute kidney injury, liver injury, pancreatitis, and DIC. Venoarterial ECMO provides an effective means of support until the recovery of organ function. Trimetazidine and magnesium are helpful in suppressing fatal arrhythmias. This report emphasizes that early recognition and early institution of ECMO can save many young lives who succumb to toxic effects of this poison.

Keywords: Aluminum phosphide, Extracorporeal membrane oxygenation, Magnesium, ST elevation myocardial infarction, Trimetazidine. Indian Journal of Critical Care Medicine (2020): 10.5005/jp-journals-10071-23533
\end{abstract}

\section{INTRODUCTION}

Aluminum phosphide is a common form of poisoning in India with no specific antidote. Refractory myocardial depression due to aluminum phosphide poisoning (ALP) toxicity carries a very high mortality rate., ${ }^{1,2}$ Patients deteriorate rapidly due to the effect of phosphine gas produced when the poison comes in contact with moisture or acid in the stomach causing a cyanide-like toxicity in which mitochondria are unable to utilize oxygen due to the inhibition of the cytochrome oxidase enzyme system leading to cellular hypoxia. ${ }^{3}$ The resultant anaerobic metabolism and severe lactic acidosis causes multiorgan dysfunction and death ensues. ${ }^{3}$ Extracorporeal membrane oxygenation (ECMO) has shown consistently positive results by providing cardiovascular support till the poison is eliminated from the body. ${ }^{11}$ Trimetazidine preserves oxidative metabolism and magnesium by stabilizing cardiac membrane and decreases arrhythmias. ${ }^{17,18}$

\section{Case Description}

A 25-year-old man without any comorbidities was brought to emergency department with sudden onset of dyspnea. He was hypotensive or arrival not responding to intravenous fluids and high-dose vasopressors. Arterial blood gas (ABG) showed severe metabolic acidosis with electrocardiograph (ECG) showing ST elevation in lateral leads. Two-dimensional (2D) echocardiography
${ }^{1}$ Department of Intensive Care, Apollo Hospitals, Bengaluru, Karnataka, India

${ }^{2}$ Department of Critical Care, Apollo Hospitals, Bengaluru, Karnataka, India

Corresponding Author: Gunavanthi Jayakumar Himaaldev, Department of Intensive Care, Apollo Hospitals, Bengaluru, Karnataka, India, Phone: +91 9901528972, e-mail: himaldev@yahoo.co.in

How to cite this article: Rao CC, Himaaldev GJ. STEMI in Young Befogged by Aluminum Phosphide Toxicity-Role of ECMO as Salvage Therapy and Trimetazidine and Magnesium to Suppress Arrhythmias. Indian J Crit Care Med 2020;24(8):727-730.

Source of support: Apollo Hospitals, Seshadripuram

Conflict of interest: None

(echo) revealed global left ventricular dysfunction, ejection fraction of $25 \%$ along with elevation of cardiac biomarkers. His condition rapidly deteriorated with refractory hypotension and severe lactic acidosis. Initiated on venoarterial (VA) ECMO as a rescue therapy with left femoral venous access $(25 \mathrm{Fr})$ and right femoral arterial access (17 Fr) with an extra $6 \mathrm{Fr}$ sheath inserted distally into right femoral artery for the perfusion of distal leg cannula. Pump flow was maintained at $4.5 \mathrm{~L} /$ minute. Heparin-bonded circuit was used and anticoagulation was avoided due to coagulopathy. He progressed to 


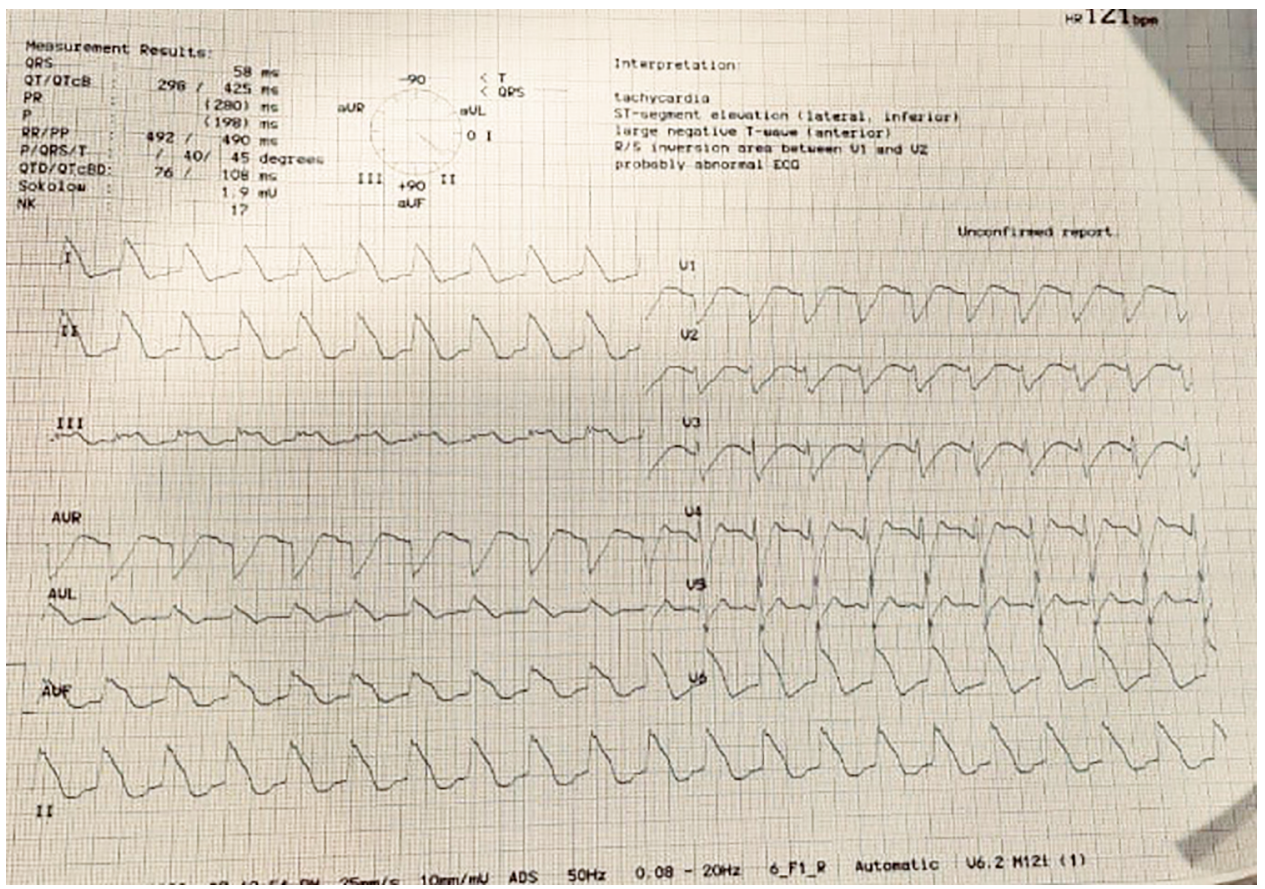

Fig. 1: ECG changes showing broad QRS complexes

acute kidney injury with worsening of lactic acidosis and developed wide QRS complexes (Fig. 1) and he was treated with magnesium and trimetazidine. He also developed severe ischemic hepatitis with disseminated intravascular coagulation (DIC) and was managed with blood product transfusion. The serum transaminases peaked at day 3 and showed a decreasing trend by day 5 . His pancreatic enzymes were also elevated and a diagnostic ultrasound confirmed pancreatitis which was managed conservatively. Dobutamine was initiated as the vasopressors gradually tapered with good clearance of lactate and good peripheral perfusion. Serial 2D echo showed improvement in left ventricle (LV) function. He was continued on dialysis because of oliguria and recurrent pulmonary edema. Patient was weaned off VA ECMO successfully on day 6 and extubated onto high-flow nasal cannula on day 7. His liver and renal functions gradually recovered and coagulopathy reversed and he was discharged home on day 12 (Tables 1 and 2).

\section{Discussion}

Aluminum phosphide is a common suicidal agent in India and associated with very high mortality of about $80-90 \%$. Aluminum phosphide poisoning can have a varied presentation and clinical course. The initial symptoms can be non-specific and include epigastric pain, vomiting, diarrhea, dizziness, and dyspnea. ${ }^{1,2}$ The deadly phosphine gas that is generated as a result of its interaction with moisture or acid in the stomach results in inhibition of the cytochrome oxidase enzyme system in mitochondria due to which the body is unable to utilize oxygen for aerobic metabolism. ${ }^{1}$ The ultimate dependence on anaerobic metabolism results in significant lactate accumulation and severe metabolic acidosis affecting the major organ systems. Cellular damage and hypoxia causes cardiotoxicity, resistant hypotension, lung injury, and liver failure which are the most common causes of mortality and morbidity after its poisoning. Cardiovascular complications including dysrhythmias, such as ventricular tachycardia and systolic heart failure, ranging from decreased cardiac function to complete cardiovascular collapse are common in ALP. ${ }^{3-6,10,11}$ The initial ECG for our patient showed ST elevation similar to acute presentations of myocarditis. He developed wide QRS complexes on day 2 of ECMO which was managed by magnesium, trimetazidine, and afterload reduction with nitrates.

Trimetazidine enhances glucose oxidation by inhibiting beta-oxidation of fatty acids, thus preventing further insult to the myocardium. ${ }^{18}$ Lidocaine, magnesium, and amiodarone have been used to convert ventricular tachycardia to sinus rhythm in patients with ALP toxicity. ${ }^{4,7}$ Magnesium acts as a cell membrane stabilizer in the myocardium and combats free radical stress due to phosphine. ${ }^{17}$ Since there is no specific antidote available, ECMO has shown promise by acting as a rescue therapy providing cardiovascular support until the effect of the poison wears off and the end organs recover. ECMO has been well described for reversible cardiogenic shock in patients with ALP. ${ }^{3,8-11}$ Hemodialysis has been described as treatment for acute renal failure and metabolic acidosis in ALP. ${ }^{13-15} \mathrm{~N}$-Acetyl cysteine, which was administered to our patient, has been described as treatment for cardiotoxicity secondary to oxidative stress in ALP and its use has been associated with a decreased hospital length of stay. ${ }^{12}$ Acute pancreatitis and ischemic hepatitis are known complications and improved with cardiovascular support with ECMO. In our case, ECMO was initiated early for cardiovascular support in view of severe myocarditis and hemodynamic instability as the initial presentation was ST elevation myocardial infarction (STEMI) and severe left ventricular dysfunction. A high index of suspicion for ALP is necessary in these clinical presentation given the potential for rapid progression of multisystem organ failure, cardiovascular collapse, and death. 2,10,16

\section{Conclusion}

Aluminum phosphide poisoning has varied presentation and can be confused with cardiac emergency because of the ECG changes and myocardial dysfunction. There is no specific antidote for ALP and there is high risk of multiorgan failure resulting in acute kidney injury, liver injury, pancreatitis, and DIC with high mortality. 
Table 1: Key laboratory variables with time course of illness

\begin{tabular}{|c|c|c|c|c|c|c|c|c|}
\hline Complete blood count & Day 1 & Day 2 & Day 3 & Day 4 & Day 5 & Day 6 & Day 7 & Day 8 \\
\hline Hemoglobin (g/dL) & 15.9 & 8.8 & 8.3 & 9.1 & 9.8 & 9.0 & 9.2 & 10 \\
\hline Total WBC count $\left(10^{9} / \mathrm{L}\right)$ & 11.7 & 10.5 & 4.7 & 4.4 & 6.6 & 12.2 & 14 & 13.6 \\
\hline Platelets $\left(10^{9} / \mathrm{L}\right)$ & 324 & 72 & 89 & 83 & 65 & 80 & 85 & 125 \\
\hline \multicolumn{9}{|l|}{ Renal function tests } \\
\hline Sodium (mEq/L) & 137 & 144 & 144 & 143 & 141 & 138 & 140 & 138 \\
\hline Potassium (mEq/L) & 4 & 4.4 & 4.1 & 4.6 & 4.1 & 4.7 & 4.3 & 3.5 \\
\hline Urea (mg/dL) & 24 & 30 & 127 & 144 & 85 & 183 & 144 & 62 \\
\hline Creatinine $(\mathrm{mg} / \mathrm{dL})$ & 1.1 & 1.3 & 5.1 & 5.3 & 3.3 & 6 & 4.8 & 2.1 \\
\hline \multicolumn{9}{|l|}{ Ischemic hepatitis } \\
\hline Total bilirubin (mg/dL) & 0.7 & 1.6 & 2.0 & 2.2 & 2.1 & 1.9 & & \\
\hline Alanine transaminase (U/L) & 31 & 1,152 & 7,515 & 5,310 & 1,838 & 441 & 841 & 76 \\
\hline Aspartate transaminase (U/L) & 33 & 1,051 & 3,385 & 2,590 & 1,884 & 1,377 & 166 & 366 \\
\hline Prothrombin time (seconds) & 14.7 & 24.3 & 22.3 & 19.3 & 15.4 & 13.4 & 15.4 & 20.5 \\
\hline International normalized ratio & 1.12 & 1.91 & 1.74 & 1.5 & 1.18 & 1.02 & 1.18 & 1.59 \\
\hline Activated prothrombin time (seconds) & 23.9 & 40.9 & 37.7 & 47.8 & 36 & 27.7 & 27 & 27 \\
\hline Fibrinogen (mg/dL) & 270 & 98 & 110 & & 170 & & & 293 \\
\hline \multicolumn{9}{|l|}{ Acute pancreatitis } \\
\hline Amylase (IU/L) & & 1,120 & & & 987 & & & \\
\hline Lipase (IU/L) & & 174.3 & & & 289.6 & & & \\
\hline
\end{tabular}

Table 2: Key clinical parameters with time course of illness

\begin{tabular}{|c|c|c|c|c|c|c|c|c|}
\hline & Day 1 & Day 2 & Day 3 & Day 4 & Day 5 & Day 6 & Day 7 & Day 8 \\
\hline Ejection fraction & $25 \%$ & $30 \%$ & $35 \%$ & & $40 \%$ & & $50 \%$ & \\
\hline Lactate levels (mmol/L) & 8.8 & 4.6 & 2.5 & 2.8 & 1.7 & 1.4 & 1.6 & 1.2 \\
\hline
\end{tabular}

Trimetazidine and magnesium help to suppress arrhythmia. Venoarterial ECMO provides an effective means of cardiovascular support until the poison is eliminated from the body. Our case emphasizes that early recognition and early institution of ECMO can save many young lives who succumb to toxic effects of this poison.

\section{Clinical Significance}

A high index of suspicion is required for ALP because of its confusing presentation similar to cardiac emergencies, given the potential for rapid progression and severe multiorgan toxicity. Fatal arrhythmias are common and can be successfully treated with trimetazidine and magnesium. Early recognition and early institution of ECMO is the key to a successful outcome.

\section{ACKNoWledgments}

We acknowledge cardiothoracic surgeons, anesthetists, perfusionists, nurses, and respiratory therapist for their valuable support.

\section{References}

1. Shadnia S, Sasanian G, Allami P, Hosseini A, Ranjbar A, Amini-Shirazi $\mathrm{N}$, et al. A retrospective 7 years study of aluminum phosphide poisoning in Tehran: opportunities for prevention. Hum Exp Toxicol 2009;28(4):209-213. DOI: 10.1177/0960327108097194.

2. Sharma A, Dishant VG, Kaushik JS, Mittal K. Aluminum phosphide (celphos) poisoning in children: a 5 year experience in a tertiary care hospital from northern india. Indian J Crit Care Med 2014;18(1):33-36. DOI: 10.4103/0972-5229.125434.
3. Elabbassi W, Chowdhury MA, Fachtartz AA. Severe reversible myocardial injury associated with aluminium phosphide toxicity: a case report and review of literature. Journal of the Saudi Heart Association 2014;26(4):216-221. DOI: 10.1016/j.jsha.2013.11.006.

4. Siwach SB, Singh $H$, Katyal VK, Bhardwaj G. Cardiac arrhythmias in aluminium phosphide poisoning studied by on continuous holter and cardioscopic monitoring. J Assoc Phys India 1998;46(7):598-601.

5. Soltaninejad K, Beyranvand MR, Momenzadeh SA, Shadnia S. Electrocardiographic findings and cardiac manifestations in acute aluminum phosphide poisoning. J Forensic Leg Med 2012;19(5): 291-293. DOI: 10.1016/j.jflm.2012.02.005.

6. Akkaoui M, Achour S, Abidi K, Himdi B, Madani A, Zeggwagh AA, et al. Reversible myocardial injury associated with aluminum phosphide poisoning. Clin Toxicol 2007;45(6):728-731. DOI: 10.1080/15563650701517350.

7. Chugh SN, Malhotra S, Kumar P, Malhotra KC. Reversion of ventricular and supraventricular tachycardia by magnesium sulphate therapy in aluminium phosphide poisoning. report of two cases. J Assoc Phys India 1991;39(8):642-643.

8. Mohan B, Gupta V, Ralhan S, Gupta D, Puri S, Wander GS, et al. Role of extracorporeal membrane oxygenation in aluminum phosphide poisoning-induced reversible myocardial dysfunction: a novel therapeutic modality. J Emerg Med 2015;49(5):651-656. DOI: 10.1016/j.jemermed.2015.06.071.

9. Mohan B, Singh B, Gupta V, Ralhan S, Gupta D, Puri S, et al. Outcome of patients supported by extracorporeal membrane oxygenation for aluminum phosphide poisoning: an observational study. Indian Heart J 2016;68(3):295-301.

10. Merin O, Fink D, Fink DL, Shahroor S, Schlesinger Y, Amir G, et al. Salvage ECMO deployment for fatal aluminum phosphide poisoning. Am J Emerg Med 2015;33(11):1718. DOI: 10.1016/j.ajem.2015.03.054.

11. Lehoux J, Hena Z, McCabe M, Peek G. Aluminium phosphide poisoning resulting in cardiac arrest, successful treatment with 
extracorporeal cardiopulmonary resuscitation (ECPR): a case report. Perfusion 2018;33(7):597-598. DOI: 10.1177/0267659118777196.

12. Tehrani H, Halvaie Z, Shadnia S, Soltaninejad K, Abdollahi M. Protective effects of $\mathrm{N}$-acetylcysteine on aluminum phosphideinduced oxidative stress in acute human poisoning. Clin Toxicol 2013;51(1):23-28. DOI: 10.3109/15563650.2012.743029.

13. Bayazıt AK, Noyan A, Anarat A. A child with hepatic and renal failure caused by aluminum phosphide. Nephron 2000;86(4):517. DOI: 10.1159/000045849.

14. Nasa P, Gupta A, Mangal K, Nagrani SK, Raina S, Yadav R. Use of continuous renal replacement therapy in acute aluminum phosphide poisoning: a novel therapy. Ren Fail 2013;35(8):1170-1172. DOI: 10.3109/0886022X.2013.815565.

15. Bashardoust B, Farzaneh E, Habibzadeh A, Sadeghi MS. Successful treatment of severe metabolic acidosis due to acute aluminum phosphide poisoning with peritoneal dialysis: a report of 2 cases. Iran J Kidney Dis 2017;11(2):165.

16. Yan H, Chen H, Li Z, Shen M, Zhuo X, Wu H, et al. Phosphine analysis in postmortem specimens following inhalation of phosphine: fatal aluminum phosphide poisoning in children. J Anal Toxicol 2018;42(5):330-336. DOI: 10.1093/jat/bky005.

17. Chugh SN, Kamar P, Sharma A, Chugh K, Mittal A, Arora B. Magnesium status and parenteral magnesium sulphate therapy in acute aluminum phosphide intoxication. Magnes Res 1994;7(3-4): 289-294.

18. Dueñas A, Pérez-Castrillon JL, Cobos MA, Herreros V. Treatment of the cardiovascular manifestations of phosphine poisoning with trimetazidine, a new antiischemic drug. Am J Emerg Med 1999;17(2):219-220. DOI: 10.1016/S0735-6757(99) 90075-X. 